\title{
In-Pavement Fiber Bragg Grating Sensor for Vehicle Counting
}

\author{
Mu'ath Al-Tarawneh \\ muath.altarawneh@mutah.edu.jo \\ Department of Civil and Environmental Engineering, Mu'tah University, Karak, Jordan \\ Ying Huang \\ ying.huang@ndus.edu \\ Department of Civil and Environmental Engineering, North Dakota State University, Fargo, North Dakota, \\ USA
}

\begin{abstract}
Traffic volume studies are conducted to determine the number, movements, and classifications of roadway vehicles at a given location and period. Typically, there are two methods for conducting traffic volume studies: manual and automatic counting. When manual counting is used, a person records the traffic volume on the site or alternatively from video recordings and this estimate can have a large margin of error. Automatic counting is based on measurement technologies, including pneumatic road tubes, inductive loops, infrared, microwave Doppler/radar, passive acoustic, video image detection, and Bluetooth devices. However, they are costly to install and have various limitations, such as high maintenance cost, availability of power source, and dependence on surrounding environment. Currently, weigh-in-motion (WIM) technology has become popular for automatic vehicle counting. In this paper, a three-dimensional glass fiber-reinforced polymer packaged fiber Bragg grating sensor (3-D GFRP-FBG) is introduced for in-pavement vehicle counting. The 3D GFRP-FBG sensor was installed on I-94 freeway, at MnROAD facility, Minnesota. When a vehicle passes over the road, the pavement produces strain signals that are picked up by wavelength changes. These strain peaks can be tracked to achieve vehicle counting. The sensors were laid out 9 feet from the road centerline with 16 feet distance between them to detect all the vehicles travelling on the right side of the road. The feasibility tests show the ability of the sensors to detect vehicles from small cars to semi tractor-trailer. For a 250 -second period, the sensor detected 23 vehicles, with a total of 69 axles.
\end{abstract}

Keywords: Fiber Bragg grating sensor; Vehicle identification; Traffic monitoring; Glass fiber reinforced polymer

\section{INTRODUCTION}

Accurate traffic volume estimations on various road segments are critical to the appropriate roadway features' geometric design, traffic demand planning, and administrative purposes. In addition, Traffic volume data are essential in many transportations and decision-making models. They are used to estimate vehicle miles travelled (VMT), evaluation of infrastructure management needs such as roadway geometric improvement and maintenance scheduling (Apronti et al., 2016).

Vehicle counting can be defined as the activity of measuring and recording traffic characteristics such as vehicle volume, classification, speed, weight, or a combination 
of these characteristics (FHWA, 2013). Typically, there are two methods for counting traffic: manual and automatic counting. When manual counting is used, a person records the traffic volume including vehicle counts at intersections which is a turning movement count, estimation of average daily traffic (ADT) and annual average daily traffic (AADT) on the site or alternatively from video recordings, and the impact of the manual count error is application dependent (Zheng and Mike, 2012). Automatic counting is based on measurement technologies, including pneumatic road tubes, inductive loops, infrared, microwave Doppler/radar, passive acoustic, video image detection, and Magnetometer (Mimbela and Kelin, 2000). The existing automatic counting technologies suffer from the following disadvantages (FHWA, 2013), (Kown, 2003):

1. High cost: Most of the existing technologies require expensive instruments and need significant maintenance and calibration and they are costly to install.

2. Energy efficiency: Most of the existing technologies need to be connected to a power source or a battery in a constant manner.

3. Large scale effectiveness: Majority of the existing technologies cannot be deployed on large scale due to limitations, such as availability of energy sources and high maintenance and installation cost.

4. Stability: Most of the existing technologies show significant dependence on surrounding environment.

5. Telecommunication infrastructure: Most of the existing technologies require a telecommunication platform to transfer data to the diagnose system in order to be analyzed.

In recent decades, weigh-in-motion (WIM) technology has become popular for vehicle counting; there are several in-pavement sensors to be considered including bending plates, piezoelectric sensor, load cells, and fiber-optic sensors.

Table 1 compares the cost, accuracy, sensitivity, and life cycle for these sensors for vehicle counting. The electrical sensors (Piezoelectric sensor, bending plate and load cell) shows significant dependence on surrounding environments, such as moisture. It also shows high electromagnetic interferences (EMI) and relatively short life cycle with a moderate reliability and accuracy.

Table 1 Sensor Comparison (Majumder et al., 2008; Zhang et al., 2007; Lee, 2003)

\begin{tabular}{|c|c|l|c|c|}
\hline & Piezoelectric sensor & Bending plate & Single load cell & Optic fiber sensor \\
\hline Life cycle cost & Low $(\$ 5,000)$ & Medium $(\$ 6,000)$ & High $(\$ 8,000)$ & Low $(\$ 1,000)$ \\
\hline Accuracy & $+/-15 \%$ & $+/-10 \%$ & $+/-6 \%$ & $+/-10 \%$ \\
\hline Sensitivity & High & Medium & Low & High \\
\hline Expected life & 4 years & 6 years & 12 years & $>15$ years \\
\hline
\end{tabular}

Table 1 shows the preference of optic fiber sensor on the electrical sensors. Optic fiber sensor has high reliability and accuracy in addition to some unique advantages, such as small size, lightweight, high sensitivity, immunity to electromagnetic interference, and ability serving in harsh environments (Mall et al., 2008). Fiber Bragg grating (FBG) is 
the most common optic fiber sensor which has been widely used for civil engineering applications. However, bare FBG generally is easy to be damaged during installation because it is made up by optic fiberglass. Thus, FBG's sensor needs to be packaged before application. Nowadays, glass fiber-reinforced polymer (GFRP) material has become widely accepted for use in civil engineering applications. It provides a durable and reliable packaging alternative to steel (Oh and Sim, 2004). Hence, the GFRP material can be used to package FBG to improve its ruggedness. The three-dimensional glass fiber-reinforced polymer packaged fiber Bragg grating sensor (3D-GFRP FBG) has been introduced by the author's research team for pavement health monitoring system (Zhou et al., 2012), and low-speed and high-speed WIM measurements (Al-Tarawneh and Hunag, 2017, 2016). In this study, the 3D-GFRP FBG sensor has been applied for vehicle counting.

\section{OPERATIONAL PRINCIPLE AND SENSOR DESIGN}

\subsection{Operational Principle of FBG Sensor}

FBG is made by shedding a periodic pattern of intense ultraviolet (UV) light in order to expose the core of single mode fiber, creating a refractive index modulation so called grating. Bragg wavelength form due to reflected light from the periodic refraction change (Al-Tarawneh and Huang, 2016), which can be represented as:

$$
\lambda=2 n \Lambda
$$

Where, $\mathrm{n}$ is the effective index of refraction, and $\Lambda$ is the grating periodicity of the FBG. Bragg wavelength depends on the Brag condition and the wavelength at which this reflection occurs. Light signals at wavelengths other than the Bragg wavelength pass through and only the wavelength matched with Bragg wavelength will be reflected. Figure 1 shows a schematic of the operational principle of an FBG sensor. Due to temperature and strain dependence of the grating period, the Bragg wavelength will change as function of temperature, Te, and strain, $\varepsilon$. The strain-Bragg wavelength relationship for the FBG can be described as (Al-Tarawneh and Huang, 2016):

$$
\varepsilon=\frac{1}{\left(1-P_{e}\right)}\left(\frac{\Delta \lambda}{\lambda}-\frac{\Delta \lambda_{T e}}{\lambda_{T e}}\right)
$$

Where, Pe is the optical elasticity coefficient of the optic fiber, $\lambda$ is the center wavelength from the FBG strain sensor, and $\lambda \mathrm{Te}$ is the measured center wavelength from the FBG temperature compensation sensor. When a vehicle axle passes over the road, the pavement produces strain signals that will be picked up by wavelength changes of the embedded 3D-GFRP-FBG sensor. This peak strain signal is related to the passed axle. Thus, the sensor can be applied for vehicle counting.

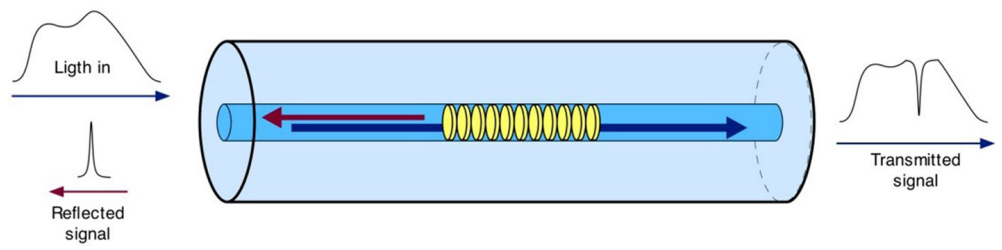

Figure 1: The Operational Principle of an FBG Sensor (Zhang et al., 2016) 


\subsection{The 3D GFRP-FBG Sensor Geometric Design}

Figure $2(\mathrm{a} \sim \mathrm{c})$ shows the geometric design for the 3D GFRP-FBG sensor. The short-gauged component of the sensor intends to detect the vertical strain while the long-gauged component is used to detect the longitudinal and transverse strains. The longitudinal direction is defined as the direction along the wheel path of the road and the transverse direction is defined as the one along the cross-section of the road. All the three components of the 3D GFRP-FBG sensor share the same diameter of 0.2 in $(5 \mathrm{~mm})$. The center wavelength of the longitudinal, transverse and vertical gauges in the 3D GFRPFBG sensor are $1529.138 \mathrm{~nm}, 1534.518 \mathrm{~nm}$, and $1524.920 \mathrm{~nm}$, respectively.

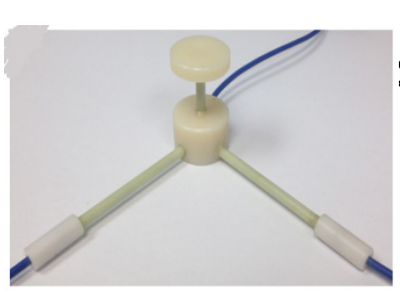

(a)

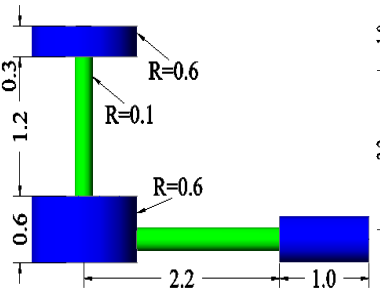

(b)

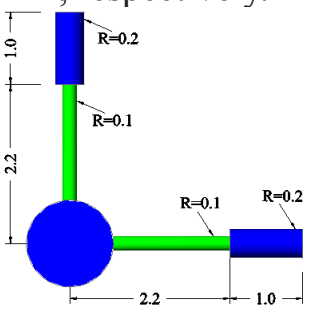

(c)

Figure 2: Geometric Design of The 3D GFRP-FBG Sensor:(a) Photo of The 3D GFRPFBG Sensor, (b) Elevation View, and (c) Plan View (Unit: in.).

\section{SENSOR FEASIBILITY TESTS}

In order to validate the feasibility of the 3D GFRP-FBG sensor for vehicle counting, field-testing was performed at the Cold Weather Road Research Facility in Minnesota (MnROAD) facility of Minnesota Department of Transportation (MnDOT). MnROAD consists of two unique roadways: a two-lane low-volume loop that is loaded with a 5-axle 80 kips $(36,287 \mathrm{~kg})$ semi-truck and a section of interstate I-94 "mainline" that contains two westbound lanes with live traffic. The 3D GFRP-FBG sensor was installed inside the pavements of Cell 17, which belongs to the interstate I-94 "mainline" westbound lanes as shown in Figure 3 (a). The 3D GFRP-FBG sensor was installed beneath the wheel path on the asphalt pavement as shown in Figure 3 (b), and in Figure 3 (c) for the photo of the sensor installation scene.

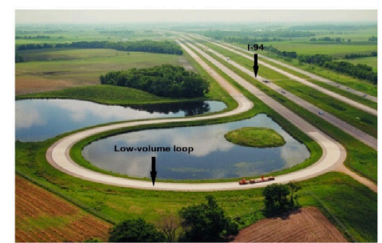

(a)

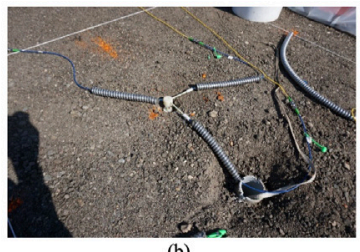

(b)

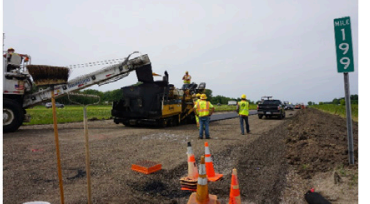

(c)

Figure 3: (a) MnROAD Facility, (b) The Sensor Installation Scene, and (c) Installation Scene 
Figure 4 shows the detail of sensors layout in Pavement Cell 17 of I-94 at MnROAD, two 3D GFRP-FBG sensors were installed under the expected wheel path, which was 9 feet from the center line of the road. The distance between the two sensors was chosen to be 16 feet in order to detect all the vehicles travelling on the right side of the road (right lane). The temperature compensation FBG sensor was installed to pick up the variation of temperature inside Pavement Cell 17, which is used in Equation 2.

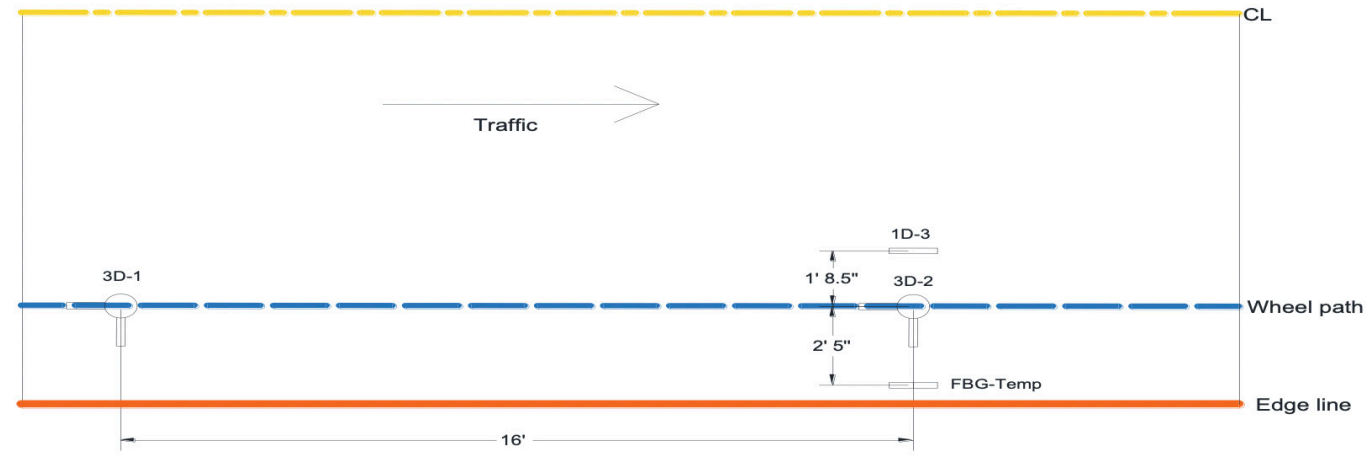

Figure 4: Sensors Layout (3D: Three Dimensions, 1D: One Dimension, CL: Center Line, 1': foot, 1": inch)

After the installation of the sensor, the three components of the sensor were connected to a $5 \mathrm{KHz}$ sampling rate $\mathrm{FBG}$ integrator together with a temperature compensation FBG sensor. The FBG integrator was further connected to a personal computer to record the data. The performed sensitivity study in previous study (Al-Tarawneh and Huang, 2016) shows that the longitudinal component has the largest weigh-in motion (WIM) measurements sensitivity among the other components, followed by the vertical component. Thus, both the longitudinal and vertical components are used in this study for vehicle counting.

Figure 5 shows the sensor's responses on its vertical and longitudinal components for a range of vehicle categories including passenger car, two-axle vehicle, three-axle vehicle, and five-axle vehicle. The average speed range for the identified vehicles was between $65 \mathrm{mph}$ and $75 \mathrm{mph}$. It is obvious that the vertical and longitudinal components responses show the ability of the sensor to detect each axle of the traveled vehicle with different responses proportionate to the tire weight. In addition, over 3 months of testing, the sensors functioning on the I-94 freeway did not appear to have any deterioration in performance. Figure 6 shows the sensor's response on its longitudinal component for a 250 -second monitoring period. During this period, 23 vehicles passed over the sensor, with a total of 69 axles. The counting of axles and vehicles was done by tracking the peaks through the sensor's response. 

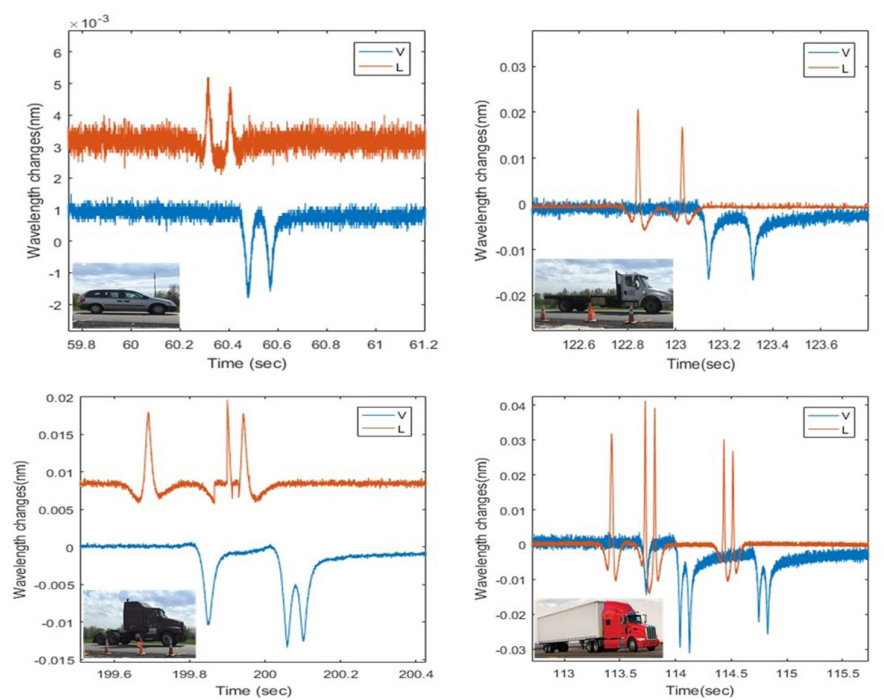

Figure 5: The Responses from Vertical and Longitudinal Components for a Range of Vehicle Categories (V: Vertical, L: Longitudinal)

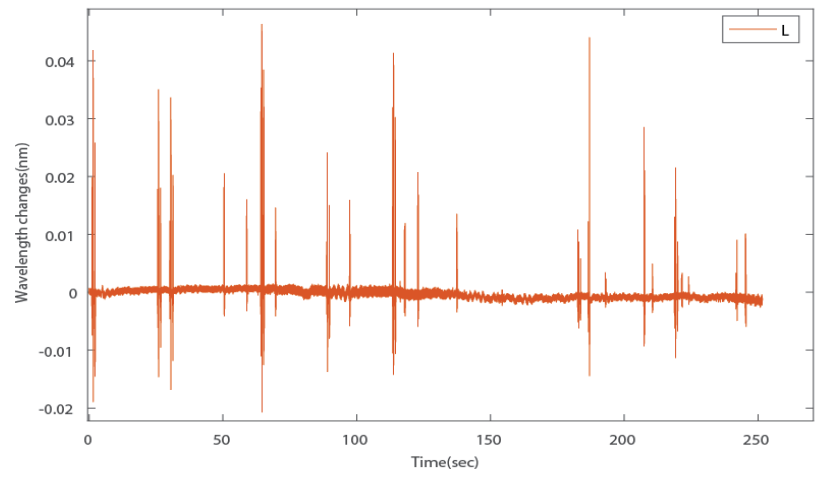

Figure 6: The Responses from Longitudinal Component (L: Longitudinal)

\section{CONCLUSION}

This study introduced a 3D GFRP FBG sensor for long term and cost-effective vehicle counting. Both of the vertical and longitudinal components of the sensor can be used for vehicle counting, since they have the largest WIM measurements sensitivity. The 3D-GFRPFBG sensors effectively monitored the travelled vehicles on the I-94 freeway, at MnROAD facility, Minnesota. It is obvious that the vertical and longitudinal components responses show the ability of the sensor to detect each axle of the travelled vehicle with different responses proportionate to the tire weight. Vehicle counting can be achieved by tracking the signal peaks through the sensor's response. Vehicles detected include size and weight ranges from small cars to tractor-trailer semi. Future efforts will endeavor to estimate vehicle speeds and to classify vehicles depending on the number of axles and the wheel base. Furthermore, Average Annual Daily Truck Traffic (AADTT) can be calculated since the sensor response appears to be closely proportional to the vehicle weight, which allows the sensor to distinguish truck from a small vehicle. 


\section{REFERENCES}

Al-Tarawneh, Mu'ath. \& Huang, Ying (2017). In-pavement fiber Bragg grating sensors for highspeed weigh-in- motion measurements. In Sensors and Smart Structures Technologies for Civil, Mechanical, and Aerospace Systems, vol. 10168, p. 101681Y. International Society for Optics and Photonics.

Al-Tarawneh, Mu'ath. \& Huang, Ying. (2016). Glass fiber-reinforced polymer packaged fiber Bragg grating sensors for low-speed weigh-in-motion measurements. Optical Engineering, Vol. 55, No. 8, 086107.

Apronti, Dick, Ksaibati, Khaled, Gerow, Kenneth \& Hopner, Jaime. (2016). Estimating traffic volume on Wyoming low volume roads using linear and logistic regression methods. Journal of traffic and transportation engineering (English edition) 3, no. 6 (2016): 493-506.

Federal Highway Administration (FHWA) (2013). Traffic Monitoring Guide. Washington, D.C.

Kwon, J., Varaiya, P. \& Skabardonis A. Estimation of truck traffic volume from single loop detectors with lane-to-lane speed correlation. Transportation Research Record: Journal of the Transportation Research Board, Vol. 1856, 2003, pp. 106-117.

Lee, Byoungho. (2003). Review of the present status of optical fiber sensors. Optical Fiber Technology, Vol. 9, No. 2, pp. 57-79.

Majumder, Mousumi, Gangopadhyay, Tarun, Chakraborty, Ashim, Dasgupta, Kamal \& Bhattacharya, Dipak. (2008). Fibre Bragg gratings in structural health monitoring-Present status and applications. Sensors and Actuators A: Physical, 147, No. 1, pp. 150-164.

Malla, Ramesh, Sen, Amlan \& Garrick Norman. (2008). A special fiber optic sensor for measuring wheel loads of vehicles on highways. Sensors, Vol. 8, No. 4, pp. 2551-2568.

Mimbela, Luz Elena \& Kelin Lawrence. (2000). Summary of vehicle detection and surveillance technologies used in intelligent transportation systems.

Oh, Hs. \& Sim, J., (2004). Interface debonding failure in beams strengthened with externally bonded GFRP. Composite Interfaces, Vol. 11, No. 1, pp. 25-42.

Zhang, Lixin, Haas, Carl \& Tighe, Susan. (2007). Evaluating weigh-in-motion sensing technology for traffic data collection. (Annual Conference of the Transportation Association of Canada), pp. 1-17.

Zhang, Qinghua, Wang, Yuan, Sun, Yangyang, Gao, Lei, Zhang, Zhenglin, Zhang, Wenyuan, Zhao P \& Yue Y. (2016). Using custom fiber Bragg grating-based sensors to monitor artificial landslides. Sensors, 16(9):1417.

Zheng, Penguin \& Mike, McDonad. (2012). An Investigation on the Manual Traffic Count Accuracy. Procedia - Social and Behavioral Sciences, Vol. 43, 2012, pp. 226-231.

Zhou, Zhi, Liu, Wanqiu, Huang, Ying, Wang, Huaping, Jianping, He, Huang, Minghua, \& Jinping, Ou. (2012). Optical fiber Bragg grating sensor assembly for 3D strain monitoring and its case study in highway pavement. Mechanical Systems and Signal Processing, Vol. 28, pp. 36-49. 